\title{
Letters from Iceland
}

\author{
The largest-ever set of human genomes from a single population and the consequent insights into mutation, \\ evolution, gene function and disease predisposition are reported in four linked papers. These results provide a \\ strategy for the analysis of the full spectrum of genetic variation in any population and raise questions about how \\ society should implement the knowledge gained.
}

$\mathrm{K}$ ari Stefansson and colleagues from deCODE Genetics have determined the sequence of the whole genomes of 2,636 Icelanders and imputed the variants found into the rest of the population via over 104,000 genotyped individuals and the pedigrees of the Icelandic people (Nat. Genet. doi:10.1038/ng.3247; 25 March 2015). Using these data, they have identified mutations in $A B C A 7$ that lead to an increased risk of Alzheimer's disease in Iceland and other populations of European ancestry (Nat. Genet. doi:10.1038/ ng.3246; 25 March 2015). They have also identified 1,171 genes knocked out in a homozygous or compound heterozygous state in live individuals (Nat. Genet. doi:10.1038/ng.3243; 25 March 2015). Knockout humans were found at a prevalence of $7.7 \%$ among the genotyped population, and at least some of the underlying variants were found by a transmission calculation to be deleterious and likely under selection. The Y-chromosome sequences of 753 Icelandic men offer insight into our mode of evolution through accurate measurements of the different rates of mutation and gene conversion in unique and repeat regions on the Y chromosome (Nat. Genet. doi:10.1038/ng.3171; 25 March 2015).

Not all of the genetic variants found in this research program will be equally informative, but some of the early findings have offered clues about disease mechanisms or pointed the way to translational options in medicine. For example, the rare $A P P$ variant c.673A $>\mathrm{T}$ (rs63750847[A]) that is protective against Alzheimer's disease provides a model suggesting that treatments to reduce proteolysis at the $\beta$ cleavage site of the encoded protein may be effective against neurodegeneration and disease progression (Nature 488, 96-99, 2012; doi:10.1038/nature11283). A rare deletion found in individuals with schizophrenia confers alterations in brain structure and learning difficulties with reading and mathematical calculation in carriers without a diagnosis of schizophrenia (Nature 505, 361-366, 2014; doi:10.1038/nature12818). This study may point the way to how genetic differences in the brain affect learning style, the way different people make use of educational experiences and how people with genetically different cognitive approaches interact with other environmental stressors that can push a vulnerable person into the disease state.

Among Iceland's population (323,002 in 2013), there are over 2,000 men and women who carry a loss-of-function mutation in $B R C A 2$ encoding p.Asn257Lysfs ${ }^{*} 17$ that confers a 4.6 -fold increase in the lifetime risk of developing any cancer. The probability of particular types of cancer associated with this mutation is notable. At least 724 of the women will develop cancer of the breast or ovary, and at least 360 of the men will develop cancer of the prostate. From the epidemiological data of Icelanders born between 1914 and 1934, the mutation results in a difference in life expectancy of 11 fewer years for a woman and 7 fewer years for a man. Another mutation, c.2040_2041insTT in BRIP1, found at an allele frequency of $0.41 \%$, confers an elevated risk of ovarian cancer and is associated with an overall reduction of 3.6 years in lifespan for all cancers (Nat. Genet. 43, 1104-1107, 2011; doi:10.1038/ng.955).

Because the deCODE genomic projects were consented for research, it would be ethically inappropriate for the researchers to contact the mutation-carrying individuals via their physicians. But, as enhanced cancer screening at younger ages than usually recommended for the general population can influence the health and life expectancy outcomes of mutation carriers and their relatives who also carry the mutation, to do nothing at all would also be ethically wrong. In our view, the decision lies with the Icelanders themselves once they have been given the information about the number of people at risk of these diseases and the options available. One way in which they can see how others have used such information is through Joanna Rudnick's thoughtful documentary In the Family about the decisions facing the carriers of a similar cancer-predisposing mutation in the BRCA1 gene (http://inthefamily.kartemquin.com/).

The researchers have offered a unique and detailed portrait of the genetic variation in their population. It is now time for those in the population and around the world to help transform these findings into useful medical and cultural knowledge according to existing values and the changed priorities resulting from the new information. This handover from scientific curiosity to utility driven by community values is prefigured in the poem "After Reading a Child's Guide to Modern Physics" by W.H. Auden, a man greatly influenced by his interactions with Iceland and its people: "This passion of our kind/ For the process of finding out/ Is a fact one can hardly doubt,/ But I would rejoice in it more/ If I knew more clearly what/ We wanted the knowledge for."

We thank Illumina for sponsorship of this Focus on Genomes of Icelanders. 